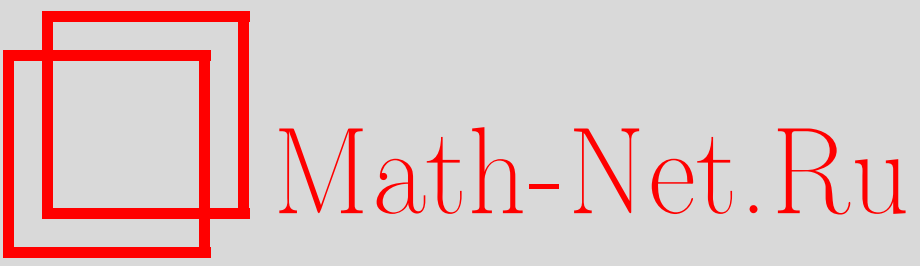

Д. Д. Холм, А. Хон, Неинтегрируемость уравнения пятого порядка с интегрируемой динамикой двух тел, ТМФ, 2003, том 137, номер 1, 121-136

DOI: https://doi.org/10.4213/tmf250

Использование Общероссийского математического портала Math-Net.Ru подразумевает, что вы прочитали и согласны с пользовательским соглашением

http://www . mathnet.ru/rus/agreement

Параметры загрузки:

IP : 3.89 .185 .249

26 апреля 2023 г., 13:31:13 
ТЕОРЕТИЧЕСКАЯ

И МАТЕМАТИЧЕСКАЯ

ФИЗИКА

Том 137, № 1

октябрь, 2003

(C) 2003 г.

Д. Д. Холм* , А.Н.В. Хон ${ }^{\dagger}$

\section{НЕИНТЕГРИРУЕМОСТЬ УРАВНЕНИЯ ПЯТОГО ПОРЯДКА С ИНТЕГРИРУЕМОЙ ДИНАМИКОЙ ДВУХ ТЕЛ}

Рассматривается дифференциальное уравнение в частных производных пятого порядка, представляющее собой обобщение интегрируемого уравнения Камасса-Холма и обладаюшее точными решениями в виде суперпозиции произвольного числа пульсонов, геодезическая гамильтонова динамика которых, как известно, является интегрируемой в случае двух тел $(N=2)$. Численные расчеты показывают, что пульсоны стабильны, являются доминирующими в задаче с начальными данными и рассеиваются упруго. Эти характеристики сходны с характеристиками солитонов в интегрируемых системах. Тем не менее для данного уравнения в частных производных показано отсутствие сколько-нибудь приемлемой лагранжевой или бигамильтоновой структуры, дан негативный ответ для теста Пенлеве и для метода Уолквиста-Эстебрука. Это позволяет прийти к заключению, что это уравнение неинтегрируемо.

Ключевые слова: гамильтонова динамика, неинтегрируемость, упругое рассеяние, пульсоны.

\section{1. ВВЕДЕНИЕ}

В данной работе рассматривается уравнение в частных производных (УЧП) пятого порядка

$$
u_{4 x, t}-5 u_{x x t}+4 u_{t}+u u_{5 x}+2 u_{x} u_{4 x}-5 u u_{3 x}-10 u_{x} u_{x x}+12 u u_{x}=0 .
$$

Одна из причин, по которой это уравнение вызьвает интерес, состоит в том, что оно допускает точные решения вида

$$
u=\sum_{j=1}^{N} p_{j}(t)\left(2 e^{-\left|x-q_{j}(t)\right|}-e^{-2\left|x-q_{j}(t)\right|}\right),
$$

*Theoretical Division and Center for Nonlinear Studies, Los Alamos National Laboratory, Los Alamos, NM 87545, USA. E-mail: dholm@lanl.gov

${ }^{\dagger}$ Institute of Mathematics and Statistics, University of Kent, Canterbury CT2 7NF, UK. E-mail: anwh@ukc.ac.uk 
где эволюция переменных $p_{j}$ и $q_{j}$ описывается канонической гамильтоновой динамикой, порож даемой гамильтонианом

$$
H_{N}=\frac{1}{2} \sum_{j, k=1}^{N} p_{j} p_{k}\left(2 e^{-\left|q_{j}-q_{k}\right|}-e^{-2\left|q_{j}-q_{k}\right|}\right) .
$$

Следуя терминологии работы [1], будем называть такие решения "пульсонами". Уравнения $N$-частичной динамики пульсонов эквивалентны уравнениям геодезического потока на $N$-мерном пространстве с координатами $q_{j}$ и кометрикой

$$
g^{j k}=g\left(q_{j}-q_{k}\right), \quad g(x)=2 e^{-|x|}-e^{-2|x|} .
$$

Пульсоны (1.2) представляют собой слабые решения с разрывными вторыми производными в изолированных точках.

УЧП (1.1) принадлежит семейству интегральных УЧП, исследованному в работе [1], и может быть получено из уравнения

$$
m_{t}+u m_{x}+2 u_{x} m=0, \quad u=g * m
$$

где величина $u(x, t)$ определяется через $m(x, t)$ с помощью интеграла свертки:

$$
g * m:=\int_{-\infty}^{\infty} g(x-y) m(y, t) d y .
$$

Интегральное ядро $g(x)$ выбирается в виде четной функции, и при любом ядре $g$ уравнение (1.4) принимает гамильтонову форму Пуассона-Ли:

$$
m_{t}=-\left(m \partial_{x}+\partial_{x} m\right) \frac{\delta H}{\delta m}
$$

где

$$
H=\frac{1}{2} \int m g * m d x=\frac{1}{2} \int m u d x .
$$

Любое уравнение из этого семейства допускает пульсонные решения вида

$$
u(x, t)=\sum_{j=1}^{N} p_{j}(t) g\left(x-q_{j}(t)\right)
$$

при произвольном $N$; при этом $p_{j}$ и $q_{j}$ удовлетворяют каноническим уравнениям Гамильтона

$$
\frac{d p_{j}}{d t}=-\frac{\partial H_{N}}{\partial q_{j}}=-p_{j} \sum_{k=1}^{N} p_{k} g^{\prime}\left(q_{j}-q_{k}\right), \quad \frac{d q_{j}}{d t}=\frac{\partial H_{N}}{\partial p_{j}}=\sum_{k=1}^{N} p_{k} g\left(q_{j}-q_{k}\right),
$$


которые задаются гамильтонианом

$$
H_{N}=\frac{1}{2} \sum_{j, k} p_{j} p_{k} g\left(q_{j}-q_{k}\right) .
$$

Уравнения (1.7) описывают геодезичекое движение на многообразии с кометрикой $g^{j k}=$ $g\left(q_{j}-q_{k}\right)$. Замечательный результат работы [1] состоит в том, что динамика в случае задачи двух тел $(N=2)$ оказьвается интегрируемой при произвольном выборе ядра $g$, а численные расчеты показывают, что это упругое рассеяние пульсонов доминирует в задаче с начальными данными.

В работе [1] выделены три специальных случая, а именно (с точностью до масштабных преобразований):

- $g(x)=\delta(x)$ - римановы ударные волны;

- $g(x)=1-|x|,|x|<1,-$ компактоны;

- $g(x)=e^{-|x|}-$ пиконы.

В каждом из указанных случаев как интегральное УЧП (1.4), так и соответствующая конечномерная система (1.7) оказываются интегрируемыми (для любого $N$ ). Наиболее примечательным оказывается третий случай $\left(g(x)=e^{-|x|}\right)$, который представляет собой (масштабно растянутую) функцию Грина оператора Гельмгольца, удовлетворяющую тождеству

$$
\left(1-\partial_{x}^{2}\right) g(x)=2 \delta(x) .
$$

Применяя масштабное преобразование, получим уравнение

$$
m=u-u_{x x}
$$

а уравнение (1.4) при этом является не чем иным, как УЧП для функции $u(x, t)$, а именно

$$
u_{t}-u_{x x t}-u u_{3 x}-2 u_{x} u_{x x}+3 u u_{x}=0
$$

что представляет собой бездисперсионную редукцию интегрируемого уравнения Камасса-Холма для волн на мелкой воде [2]. Пульсонные решения самого уравнения Камасса-Холма принимают форму пиконов, или заостренных солитонов, т.е.

$$
u(x, t)=\sum_{j=1}^{N} p_{j}(t) e^{-\left|x-q_{j}(t)\right|} .
$$

Уравнение пятого порядка возникает при другом выборе функции Грина. Используя тождество

$$
\left(4-\partial_{x}^{2}\right)\left(1-\partial_{x}^{2}\right) g(x)=12 \delta(x), \quad g(x)=2 e^{-|x|}-e^{-2|x|},
$$

получим, что при таком выборе функции $g$ (при подходящем масштабном преобразовании) имеет место уравнение

$$
m=u_{4 x}-5 u_{x x}+4 u
$$

и тогда уравнение (1.4) становится уравнением пятого порядка (1.1). Тем самым УЧП (1.1) может рассматриваться как естественное обобшение старшего порядка для уравнения Камасса-Холма. 


\section{2. ГАМИЛЬТОНОВ И ЛАГРАНЖЕВ ПОДХОДЫ}

В подготовленной к публикации статье [3] будет обсуждаться более общее семейство интегральных УЧП вида

$$
m_{t}+u m_{x}+b u_{x} m=0, \quad u=g * m
$$

где $b$ - произвольный параметр. Семейство уравнений (1.4) отвечает частному случаю $b=2$, в то время как недавние численные результаты [4] были получены и в случае различных значений параметра $b$. В случае пиконного ядра $g=e^{-|x|}$ с функцией $m$, задаваемой уравнением (1.8), уравнения этого класса были проверены методом асимптотической интегрируемости в работе [5], и только случаи $b=2,3$ были отмечены как потенциально интегрируемые. В случае $b=2$ интегрируемость уравнения Камасса-Холма с помошью метода обратной задачи рассеяния была уже известна [2], но свойство интегрируемости для нового уравнения

$$
u_{t}-u_{x x t}-u u_{3 x}-3 u_{x} u_{x x}+4 u u_{x}=0
$$

с параметром $b=3$ было доказано в работе [6] с помошью конструкции пары Лакса. Два указанных случая интегрируемости $b=2,3$ были недавно выделены также с помошью подхода пертурбативных симметрий [7]. При любом $b \neq-1$ семейство пиконных решений уравнения (2.1) c $g=e^{-|x|}$ возникает как бездисперсионный предел во втором порядке асимптотического разложения уравнения волн на мелкой воде [8].

Другая причина интереса к уравнению пятого порядка (1.1) состоит в том, что оно может быть естественным образом представлено в терминах величины $m$ (уравнение $(1.12))$, задаваемой суперпозицией двух операторов Гельмгольца, действуюших на функцию $u$. Такая суперпозиция появляется в операторе пятого порядка

$$
B_{0}=\partial_{x}\left(4-\partial_{x}^{2}\right)\left(1-\partial_{x}^{2}\right)
$$

который, как было найдено в работе [6], задает первую гамильтонову структуру для нового интегрируемого уравнения (2.2). Это привело нас к гипотезе, что оператор $B_{0}$ должен естественным образом возникать в теории интегрируемых уравнений старших порядков, таких как уравнение (1.1).

Все уравнения вида (1.4) обладают гамильтоновой структурой Пуассона-Ли, задаваемой уравнением (1.5), но интегрируемость предполагает наличие бигамильтоновой структуры. В случае уравнения Камасса-Холма (1.9) существуют два способа задания второй гамильтоновой структуры. Первый способ состоит в исследовании законов сохранения с учетом того, что уравнение (1.9) может быть записано в виде

$$
m_{t}=\left(u u_{x x}+\frac{1}{2} u_{x}^{2}-\frac{3}{2} u^{2}\right)_{x}=\partial_{x} \frac{\delta \widetilde{H}}{\delta u}=\partial_{x}\left(1-\partial_{x}^{2}\right) \frac{\delta \widetilde{H}}{\delta m}
$$


для гамильтониана

$$
\widetilde{H}=-\frac{1}{2} \int\left(u u_{x}^{2}+u^{3}\right) d x
$$

Тождество (2.3) задает вторую гамильтонову структуру для уравнения КамассаХолма, а операторы $m \partial_{x}+\partial_{x} m$ и $\partial_{x}\left(1-\partial_{x}^{2}\right)$ образуют совместную бигамильтонову пару.

Сходным образом интегральная величина $\int m d x$ сохраняется для уравнения (1.1), где функция $m$ задается уравнением (1.12). Закон сохранения имеет явный вид:

$$
\left(u_{4 x}-5 u_{x x}+4 u\right)_{t}=-\left(u u_{4 x}+u_{x} u_{3 x}-\frac{1}{2} u_{x x}^{2}-5 u u_{x x}-\frac{5}{2} u_{x}^{2}+6 u^{2}\right)_{x}=: \mathcal{F}_{x} .
$$

По аналогии с уравнением Камасса-Холма это свойство может предполагать существование подходящего гамильтонова оператора с постоянными коэффициентами вида $\partial_{x}\left(4-\partial_{x}^{2}\right)\left(1-\partial_{x}^{2}\right)$ (который, как известно, является оператором Гамильтона для нового уравнения (2.2)). При этом правая часть уравнения (2.5) должна была бы иметь вид

$$
\partial_{x} \frac{\delta K}{\delta u}=\partial_{x}\left(4-\partial_{x}^{2}\right)\left(1-\partial_{x}^{2}\right) \frac{\delta K}{\delta m} .
$$

Однако для потока уравнения (2.5) получаем

$$
\mathcal{F} \neq \frac{\delta K}{\delta u}
$$

что справедливо для любого функционала $K$ локальной плотности от переменной $u$, и, следовательно, операторы $\partial_{x}\left(4-\partial_{x}^{2}\right)\left(1-\partial_{x}^{2}\right)$ и $m \partial_{x}+\partial_{x} m$ оказываются несовместными.

Второй способ задания гамильтоновой структуры (2.3) для уравнения КамассаХолма состоит в использовании действия (интеграла от лагранжевой плотности)

$$
S=\iint \mathcal{L}[\phi] d x d t:=\iint \frac{1}{2}\left(\phi_{x} \phi_{t}-\phi_{3 x} \phi_{t}+\phi_{x} \phi_{x x}^{2}+\phi_{x}^{3}\right) d x d t
$$

для поля $u=\phi_{x}$. Преобразование Лежандра задает сопряженный импульс

$$
\frac{\partial \mathcal{L}}{\partial \phi_{t}}=\frac{1}{2}\left(\phi_{x}-\phi_{3 x}\right)=\frac{m}{2}
$$

и тот же самый гамильтониан, что и $(2.4)$, т.е.

$$
\widetilde{H}=\int\left(\frac{1}{2} m \phi_{t}-\mathcal{L}\right) d x
$$

Если попытаться использовать этот подход к уравнению (1.1), то следует положить $u=$ $\phi_{x}$ и переписать его в виде

$$
\phi_{5 x, t}-5 \phi_{3 x, t}+4 \phi_{x t}+\phi_{x} \phi_{6 x}+2 \phi_{x x} \phi_{5 x}-5 \phi_{x} \phi_{4 x}-10 \phi_{x x} \phi_{3 x}+12 \phi_{x} \phi_{x x}=0 .
$$


Но уравнение (2.6) не может быть получено ни из какой локальной лагранжевой плотности $\mathcal{L}[\phi]$ ввиду наличия членов $\phi_{x} \phi_{6 x}+2 \phi_{x x} \phi_{5 x}$.

Первая нелокальная гамильтонова структура для уравнения Камасса-Холма может быть получена путем применения оператора рекурсии к оператору $m \partial_{x}+\partial_{x} m$. Уравнение (1.9) при этом может быть переписано в гамильтоновом виде:

$$
m_{t}=\left(m \partial_{x}+\partial_{x} m\right)\left(\partial_{x}^{3}-\partial_{x}\right)^{-1}\left(m \partial_{x}+\partial_{x} m\right) \frac{\delta \widehat{H}}{\delta m}, \quad \widehat{H}=\int m d x .
$$

С использованием того же гамильтониана $\widehat{H}$ аналогичное тождество для уравнения (1.1) примет вид

$$
m_{t}=B \frac{\delta \widehat{H}}{\delta m} \equiv\left(m \partial_{x}+\partial_{x} m\right)\left(\partial_{x}^{5}-5 \partial_{x}^{3}+4 \partial_{x}\right)^{-1}\left(m \partial_{x}+\partial_{x} m\right) \frac{\delta \widehat{H}}{\delta m},
$$

но по тем же соображениям, что и выше, следует ожидать, что формально нелокальный оператор $B$ в правой части уравнения (2.7) будет негамильтонов. В самом деле, используя выведенные в работе [3] функциональные уравнения, можно показать, что этот член не удовлетворяет тождеству Якоби.

\section{3. ОБРАТНОЕ ПРЕОБРАЗОВАНИЕ И АНАЛИЗ ПЕНЛЕВЕ}

После того как попытки найти какую-либо лагранжеву или бигамильтонову структуру для уравнения (1.1) оказались тшетными (что, впрочем, и ожидалось), можно попробовать провести тест Пенлеве для этого уравнения пятого порядка и оценить результат. Заметим тем не менее, что как уравнение Камасса-Холма (1.9), так и новое уравнение (2.2) являются примерами уравнений, удовлетворяюших слабому свойству Пенлеве [9], которое допускает алгебраические ветвления в решениях. Для этих уравнений оказалось полезным использовать обратные преобразования (см. определения в работе [10]), которые преобразуют их в уравнения с полюсными особенностями; в самом деле, именно это свойство оказалось ключевым в нашем открытии пары Лакса для уравнения (2.2) в работе [6]. Преобразования годографа этого вида ранее использовались в некоторых классах эволюционных уравнений [11], [12] для устранения ветвлений, но в данном случае речь идет о неэволюционных УЧП.

Чтобы обобшить результаты приведенного анализа, рассмотрим цельй класс уравнений

$$
m_{t}+u m_{x}+b u_{x} m=0, \quad m=u_{4 x}-5 u_{x x}+4 u
$$

( $b \neq 0$ - произвольный параметр), который представляет собой частное семейство уравнений вида (2.1), отвечаюших интегральному ядру (1.11), и включает в себя уравнение (1.1) как специальный случай при $b=2$. Каждое уравнение из класса (3.1) допускает сушествование закона сохранения

$$
\left(m^{1 / b}\right)_{t}=-\left(m^{1 / b} u\right)_{x}
$$


Таким образом, вводя новую зависимую переменную $p$ в соответствии с правилом

$$
p^{b}=-m,
$$

можно согласованным образом определить обратное преобразование к новым независимым переменным $X$ и $T$, задаваемым формулами

$$
d X=p d x-p u d t, \quad d T=d t .
$$

Преобразуя производные, получим новый закон сохранения:

$$
\left(p^{-1}\right)_{T}=u_{X}
$$

Переписьвая соотношение (1.12) в терминах производных $\partial_{X}$ и используя закон сохранения (3.4) для исключения производных от $u$, получим тождество

$$
u=\frac{1}{4}\left(5-\left(p \partial_{X}\right)^{2}\right)\left(p \partial_{X}\right) p\left(p^{-1}\right)_{T}-\frac{p^{b}}{4}
$$

из которого следует, что (3.4) может быть записано как уравнение только для переменной $p$, т.е.

$$
\left(p^{-1}\right)_{T}=\left(\frac{1}{4}\left(\left(p \partial_{X}\right)^{2}-5\right) p(\ln p)_{X T}-\frac{p^{b}}{4}\right)_{X}
$$

Уравнение пятого порядка (3.6) представляет собой обратно преобразованное уравнение (3.1). Вместо того, чтобы проводить полный тест Пенлеве для преобразованного уравнения, достаточно, следуя методу работы [13], применить к редукции уравнения (3.6) вида движушейся волны тест Пенлеве для обыкновенных дифференциальных уравнений (ОДУ). Поэтому положим $p=p(z)$ и $z=X-c T$. Получающееся при этом ОДУ пятого порядка может быть дважды проинтегрировано для получения ОДУ третьего порядка

$$
\frac{5}{8}\left(\frac{p^{\prime}}{p}\right)^{2}-\frac{1}{4}\left(p^{\prime} p^{\prime \prime \prime}-\frac{1}{2}\left(p^{\prime \prime}\right)^{2}-\frac{\left(p^{\prime}\right)^{2} p^{\prime \prime}}{p}+\frac{1}{2} \frac{\left(p^{\prime}\right)^{4}}{p^{2}}\right)-\frac{1}{2 p^{2}}=\frac{c^{-1} p^{b-1}}{4(b-1)}+\frac{d}{p}+e,
$$

где $b \neq 1$, величины $d$ и $е$ суть произвольные константы, $c$ - волновая скорость, а штрих обозначает производную $d / d z$. При $b=1$ в правой части присутствует член $\ln p$, и в этом случае имеет место логарифмическое ветвление, которое немедленно исключает этот случай из числа допустимых в соответствии с тестом Пенлеве. Сходным образом ввиду наличия члена с $p^{b-1}$ все нецелые значения параметра $b$ приводят к ветвлениям и должны быть исключены.

Применим анализ Пенлеве к уравнению (3.7) при целых значениях $b \neq 0,1$. Для этого исследуем поведение лидирующего члена вида $p \sim a\left(z-z_{0}\right)^{\mu}$ при движущейся сингулярности $z_{0}$ и целых величинах показателя степени $\mu$. При всех целых $b \leqslant-2$ согласованность достигается только при $\mu=4 /(3-b)$, которое оказывается нецелым, а потому 
приводит к алгебраическому ветвлению. В специальном случае $b=-1$ возникают четыре равновесных случая с $\mu=1$, где $a$ и резонансы зависят от $c$. Мы проверили, что ни при каком из значений параметра $c$ все резонансные значения не являются целыми числами, и, следовательно, тест Пенлеве не выполняется. При остальных целых значениях $b \geqslant 2$ получим, что $\mu=1$ при $a^{2}=1$ или $a^{2}=4$. При цельх величинах $b \geqslant 4$ условие равновесия также достигается при значениях $\mu=4 /(3-b)$, которые в общем случае не являются целыми, кроме специальных значений $\mu=-4,-2,-1$ при $b=4,5,7$, соответственно. Тем самым все целые значения параметра $b$, кроме $b=2,3,4,5,7$, исключаются в соответствии с (сильным) тестом Пенлеве ввиду присутствия алгебраических ветвлений. Можно, однако, допустить наличие таких ветвлений, если использовать слабый вариант теста Пенлеве.

Рассмотрим более подробно первые две реализации условия равновесия при целых значениях $b \geqslant 2$. Если $p \sim \pm\left(z-z_{0}\right)$, то неглавное равновесие достигается при резонансах $r=-1,-1,3$. Интересно то, что для $b=2$ резонансное условие при $r=3$ не будет выполнено, препятствие состоит в члене $c^{-1}$ (для таких условий равновесия тест Пенлеве будет, следовательно, выполняться только в пределе $c \rightarrow \infty)$; однако при всех целых значениях $b \geqslant 3$ резонансное условие будет выполнено. Тем не менее при главных условиях равновесия $p \sim \pm 2\left(z-z_{0}\right)$ резонансные значения равны $r=-1,1 / 2,3 / 2$, что означает возникновение в системе алгебраического ветвления, и (сильный) тест Пенлеве тем самым дает отрицательный результат при всех значениях параметра $b$. Проверим далее, может ли быть выполнен слабый тест Пенлеве, предложенный в статье [9], если допустить разложение по степеням $\left(z-z_{0}\right)^{1 / 2}$ в условии главного равновесия. Резонансное условие будет выполняться при $r=1 / 2$, но нарушаться при $r=3 / 2$. Это означает, что такое разложение с разрешенными ветвлениями типа квадратного корня не может задавать общее решение, поскольку оно не содержит достаточного числа варьируемых параметров. Общее решение может быть достигнуто только добавлением бесконечного числа членов по степеням $\ln \left(z-z_{0}\right)$; при этом невозможно добиться выполнения свойства Пенлеве ни в какой форме. Присутствие логарифмических ветвлений как в главных, так и в неглавных условиях равновесия прямо указывает на неинтегрируемость теории.

Интересно отметить, что в случае отсутствия первого члена в правой части уравнения (3.7) (в пределе $c \rightarrow \infty$ ) это уравнение допускает точные решения в терминах тригонометрических или гиперболических функций, отвечающие редукциям первого порядка

$$
\left(p^{\prime}\right)^{2}=1+2 d p+\frac{1}{3}\left(8 e-d^{2}\right) p^{2}, \quad\left(p^{\prime}\right)^{2}=4+8 d p+\frac{8}{3}\left(2 d^{2}-e\right) p^{2} .
$$

На самом деле можно непосредственно убедиться в том, что исходное уравнение (1.1) не проходит тест Пенлеве. Дело в том, что этот тест для уравнения Камасса-Холма (1.9) выполняется при главном условии равновесия

$$
u \sim-\frac{\phi_{t}}{\phi_{x}}+a \phi^{2 / 3}+\cdots
$$


с резонансами в точках $-1,0,2 / 3$; многообразие переменной $\phi(x, t)$ при этом сингулярно, а функция $a(x, t)$ произвольна. В случае уравнения (1.1) аналогичное условие равновесия имеет вид

$$
u \sim-\frac{\phi_{t}}{\phi_{x}}+a \phi^{4 / 3}+\cdots
$$

с резонансами в точках $-1,0,4 / 3,(1 \pm \sqrt{41}) / 6$; наличие иррационального резонансного значения предполагает присутствие логарифмического ветвления.

\section{4. МЕТОД РАСШИРЕННОЙ АЛГЕБРЫ}

Хотя анализ Пенлеве и представляет собой неплохой эвристический метод, позволяющий выделять потенциально интегрируемые уравнения, он никогда не дает окончательного доказательства неинтегрируемости. Если точное определение интегрируемости состоит в наличии бесконечного набора коммутирующих симметрий теории, то, используя развитый Шабатом и др. [14] симметрийный подход, можно определить необходимые условия интегрируемости (но нельзя ни построить пару Лакса каким-либо конструктивным образом, ни линеаризовать теорию в случае, когда эти условия выполняются). Только совсем недавно [7] симметрийный подход был обобщен на случай нелокальных или неэволюционных уравнений, таких как уравнения (1.1) и (1.9). Альтернативой симметрийного подхода служит метод расширенной алгебры, предложенньй Уолквистом и Эстебруком [15]. В этом методе, которым мы будем пользоваться в дальнейшем, пара Лакса для уравнения (1.1) ишется непосредственно в форме согласованной системы линейных уравнений

$$
\Psi_{x}=U \Psi, \quad \Psi_{t}=V \Psi
$$

с подходяшими матрицами $U$ и $V$ (обычно принимаюшими значения в фундаментальном представлении полупростой алгебры Ли). Эти матрицы могут содержать зависимость от функции $u$, ее производных и от спектрального параметра. Ясное изложение данного метода, приведенное в работе [16], оказалось весьма полезным для нас.

Условием совместности системы (4.1) служит уравнение нулевой кривизны

$$
U_{t}-V_{x}+[U, V]=0
$$

и сама суть метода Уолквиста-Эстебрука состоит в том, что при заданном исходном УЧП (в данном случае (1.1)) можно использовать уравнение (4.2) для получения функциональной зависимости матриц $U$ и $V$ от переменных $u, u_{x}$ и т.д. Отрицательный результат означает, что в теории не сушествует никакой пары Лакса подходящего вида, что предполагает неинтегрируемость данного уравнения. Этот вьвод, разумеется, зависит от исходных предположений о функциональном виде матриц $U$ и $V$.

Для упрощения обозначений будем записывать производную $n$-го порядка в виде $u_{n x}=u_{n}$. Если предположить, что уравнение (1.1) может быть представлено как закон

5 Теоретическая и математическая физика, т. 137, № 1, 2003 г. 
сохранения для переменной $m$, имеюший вид (2.5), то можно искать решение в следующей подходящей форме:

$$
U=U(m), \quad V=V\left(u, u_{1}, u_{2}, u_{3}, u_{4}\right)
$$

(зависимость от спектрального параметра не указана). Используя известные представления нулевой кривизны для уравнений (1.9) и (2.2), можно далее предположить, что матрица $U$ линейно зависит от $m$, тогда

$$
U=A m+B \equiv\left(u_{4}-5 u_{2}+4 u\right) A+B
$$

где $A$ и $B$ суть постоянные матрицы (не зависяшие ни от $x$, ни от $t$, но, возможно, зависящие от спектрального параметра). Подставляя этот анзац в уравнение (4.2) и используя закон сохранения (2.5) для того, чтобы убрать прямую зависимость от временно́й производной $m_{t}$, получим

$$
\begin{aligned}
\left(-u u_{5}-2\right. & \left.u_{1} u_{4}+5 u u_{3}+10 u_{1} u_{2}-12 u u_{1}\right) A-u_{5} V_{u_{4}}-u_{4} V_{u_{3}}- \\
& -u_{3} V_{u_{2}}-u_{2} V_{u_{1}}-u_{1} V_{u}+\left(u_{4}-5 u_{2}+4 u\right)[A, V]+[B, V]=0
\end{aligned}
$$

где нижние индексы при матрище $V$ обозначают частные производные. Ни одна из матриц не содержит зависимости от $u_{5}$, а потому выражение (4.3) линейно по переменной $u_{5}$. Поэтому, в частности, коэффициент, стояший при члене с пятой производной $u_{5}$, должен обрашаться в нуль, что приводит к уравнению $V_{u_{4}}=-u A$. Непосредственно проинтегрировав его, получим

$$
V=-u u_{4} A+\Gamma\left(u, u_{1}, u_{2}, u_{3}\right),
$$

где выражение для Г пока произвольно и должно быть определено из оставшихся в формуле (4.3) членов.

На следующем этапе, подставив выражение (4.4) в формулу (4.3), получим

$$
\begin{aligned}
\left(-u_{1} u_{4}+5 u u_{3}+10 u_{1} u_{2}-12 u u_{1}\right) A-u_{4} \Gamma_{u_{3}}-u_{3} \Gamma_{u_{2}}-u_{2} \Gamma_{u_{1}}- \\
-u_{1} \Gamma_{u}+\left(u_{4}-5 u_{2}+4 u\right)[A, \Gamma]+u u_{4}[A, B]+[B, \Gamma]=0 .
\end{aligned}
$$

Коэффициент при члене $u_{4}$ должен быть равен нулю, что приводит к уравнению

$$
\Gamma_{u_{3}}=[A, \Gamma+u B]-u_{1} A
$$

которое опять-таки можно явно проинтегрировать, получив в итоге

$$
\Gamma=e^{u_{3} A} \Delta\left(u, u_{1}, u_{2}\right) e^{-u_{3} A}-u_{1} u_{3} A-u B
$$


где $\Delta$ - произвольная функция интегрирования. Выражение (4.6) в явном виде содержит действие элемента фундаментального представления $\operatorname{Ad} \exp u_{3} A=\exp \left(\operatorname{ad} u_{3} A\right)$ на $\Delta$, что должно было бы приводить к возникновению зависимости экспоненциального типа от элемента $u_{3}$ в соответствующей паре Лакса, если только не вьполнено условие $\left(\operatorname{ad} u_{3} A\right)^{n} \Delta=0$ при некотором положительном целом значении параметра $n$. Подобная экспоненциальная зависимость выглядит маловероятной, учитывая то обстоятельство, что исходное уравнение (1.1) полиномиально по функции $u$ и ее производным. Поэтому нам надо сделать предположения, запрещающие возникновение бесконечного числа ненулевых множественных коммутаторов в формуле (4.6).

Подставляя функцию Г из формулы (4.6) в члены выражения (4.5), не содержашие зависимости от $u_{4}$, и действуя элементом фундаментального представления $\operatorname{Ad} \exp \left(-u_{3} A\right)$, получим

$$
\begin{gathered}
\left(u_{2} u_{3}+5 u u_{3}+10 u_{1} u_{2}-12 u u_{1}\right) A-u_{3} \Delta_{u_{2}}-u_{2} \Delta_{u_{1}}-u_{1} \Delta_{u}+ \\
+\left(-5 u_{2}+4 u\right)[A, \Delta]+\left[e^{-u_{3} A} B e^{u_{3} A}, \Delta\right]+ \\
+\left(u_{1} u_{3}+5 u u_{2}-4 u^{2}\right) e^{-u_{3} A} C e^{u_{3} A}=0
\end{gathered}
$$

где введено обозначение $C=[A, B]$. В принципе выражение (4.7) задается бесконечным рядом по степеням $u_{3}$, каждый коэффициент которого должен обращаться в нуль. Простейшее предположение, которое можно ввести для обрезания этого ряда, состоит в том, чтобы положить

$$
[A, C]=0
$$

откуда следует

$$
\begin{aligned}
& \operatorname{Ad} e^{-u_{3} A}(B)=e^{\operatorname{ad}\left(-u_{3} A\right)}(B)=B-u_{3} C, \\
& \text { Ad } e^{-u_{3} A}(C)=e^{\operatorname{ad}\left(-u_{3} A\right)}(C)=C,
\end{aligned}
$$

и выражение (4.7) тем самым становится линейным по переменной $u_{3}$. Удачным следствием условия (4.8) является то, что коэффициент при члене первого порядка по $u_{3}$ дает

$$
\Delta_{u_{2}}=\left(u_{2}+5 u\right) A-[C, \Delta]+u_{1} C
$$

Это выражение можно проинтегрировать без всяких дальнейших предположений, получив в итоге следующее условие:

$$
\Delta=e^{-u_{2} C} E\left(u, u_{1}\right) e^{u_{2} C}+\left(\frac{1}{2} u_{2}^{2}+5 u u_{2}\right) A+u_{1} u_{2} C .
$$

Действуя на него элементом $\mathrm{Ad} \exp u_{2} C$, можно получить и остаюшиеся в выражении (4.7) члены в виде

$$
\begin{aligned}
\left(5 u_{1} u_{2}-\right. & \left.12 u u_{1}\right) A-\left(\frac{3}{2} u_{2}^{2}+4 u^{2}\right) C-u_{2} E_{u_{1}}-u_{1} E_{u}+ \\
& +\left(-5 u_{2}+4 u\right)[A, E]+\left[e^{u_{2} C} B e^{-u_{2} C}, E\right]+u_{1} u_{2}\left[e^{u_{2} C} B e^{-u_{2} C}, C\right]=0 .
\end{aligned}
$$


При этом мы опять сталкиваемся с разложением в бесконечный ряд, в этом случае - по переменной $u_{2}$. Перед тем как начать поиск дальнейших упрошаюших условий, заметим, что коэффициент при члене, линейном по переменной $u_{2}$, имеет простой вид

$$
-E_{u_{1}}+u_{1}(5 A+[B, C])-[5 A+[B, C], E]=0
$$

и может быть непосредственно проинтегрирован, что приводит к выражению

$$
E=e^{-u_{1} D} Z(u) e^{u_{1} D}+\frac{1}{2} u_{1}^{2} D, \quad D=5 A+[B, C] .
$$

Для того чтобы проанализировать оставшиеся члены в формуле (4.10), удобно ввести следуюшие комбинации величин:

$$
F=[C,[C, B]], \quad G=[D, B]
$$

Заметим, что при этом выполняются тождества

$$
[A, D]=0=[A, F], \quad[A, G]=-[C, D]=F, \quad[[B, C], D]=0 .
$$

Коэффициент при члене $u_{2}^{2}$ в формуле (4.10) теперь может быть записан в виде

$$
-\frac{3}{2} C+\frac{1}{2}[F, E]-u_{1} F=0,
$$

а для коэффициента при члене $u_{2}^{0}$ получим выражение

$$
-12 u u_{1} A-4 u^{2} e^{u_{1} D} C e^{-u_{1} D}-u_{1} Z_{u}+4 u[A, Z]+\left[e^{u_{1} D} B e^{-u_{1} D}, Z+\frac{1}{2} u_{1}^{2} D\right]=0
$$

(после подстановки $E$ из (4.11) и действия элементом $\mathrm{Ad} e^{u_{1} D}$ фундаментального представления). Нет необходимости рассматривать уравнения

$$
\left[(\operatorname{ad} C)^{n} B, E\right]-n u_{1}(\operatorname{ad} C)^{n} B=0
$$

возникаюшие для коэффициентов при членах с $u_{2}^{n}, n \geqslant 3$. Вместо этого рассмотрим коэффициент при члене с $u_{1}$ в выражении (4.14), который имеет вид

$$
-Z_{u}+[G, Z]-4 u^{2} F-12 u A=0 .
$$

Это уравнение не удается проинтегрировать непосредственно, не вводя дальнейших предположений; простейшее из них состоит в том, чтобы положить

$$
[F, G]=0
$$


откуда получим

$$
Z=e^{G u} \Theta e^{-G u}+\frac{2}{3} u^{3} F-6 u^{2} A .
$$

Теперь надо использовать оставшиеся уравнения для того, чтобы задать коммутационые соотношения для постоянных элементов $A, B, C, D, F, G, \Theta$ алгебры Ли. Если взять коэффициент при члене, пропорциональном $u_{1}^{0}$ в выражении (4.13), то можно легко увидеть, что

$$
-\frac{3}{2} C+\frac{1}{2}[F, Z]=0
$$

и с учетом формул (4.12), (4.16) и выражения (4.17) получим

$$
[F, \Theta]=3 e^{-G u} C e^{G u}
$$

откуда немедленно следует

$$
[F, \Theta]=3 C, \quad[C, G]=0
$$

Применяя тождество Якоби, можно также получить равенства

$$
0=[C, G]=[C,[D, B]]=-[B,[C, D]]-[D,[B, C]]=[B, F],
$$

которые после повторного применения тождества Якоби дают следующий результат:

$$
[C, F]=0=[D, F]
$$

Обратимся теперь к уравнению (4.13) и применим условие (4.11) для вычисления коэффициента при члене с $u_{1}$, что в итоге приводит к условию

$$
-\frac{1}{2}[F,[D, Z]]-F=0
$$

Подставляя сюда выражение для $Z$ (формула (4.17)) и вычисляя постоянное слагаемое (при члене $u^{0}$ ) в выражении (4.20), с использованием формул (4.12), (4.18) и (4.19) можно найти, что

$$
\begin{aligned}
0=-\frac{1}{2}[F,[D, \Theta]]-F & =\frac{1}{2}([\Theta,[F, D]]+[D,[\Theta, F]])-F= \\
& =-\frac{3}{2}[D, C]-F=-\frac{5}{2} F
\end{aligned}
$$

Тогда из условия $F=0$ с применением первого из уравнений формулы (4.18) следует, что $C=0$, после чего нетрудно показать, что пара Лакса (4.1) коллапсирует в тривиальное уравнение $[U, V]=0$, а условие нулевой кривизны (4.2) сведется при этом к скалярному уравнению $m_{t}=\mathcal{F}_{x}$ так же, как и в формуле (2.5). 


\section{5. ЗАКЛЮЧЕНИЕ}

Ввиду того, что УЧП пятого порядка (1.1) попадает в класс пульсонных уравнений, исследованных Фрингером и Холмом в работе [1], у этого уравнения имеются точные решения, представляющие собой прямые суперпозиции произвольного числа пульсонов (как показано на рис. 1). Эти частные $N$-пульсонные решения имеют вид, задаваемый формулой (1.2), и в соответствии с общими результатами работы [1] получаем, что в случае $N=2$ гамильтоновы уравнения (1.7), описывающие динамику задачи двух тел, оказываются интегрируемыми. Тем не менее, применив несколько различных подходов, мы получили весьма серьезные указания на то, что УЧП пятого порядка (1.1) не интегрируемо в смысле возможного наличия пары Лакса, т.е. с помощью метода обратной задачи рассеяния.
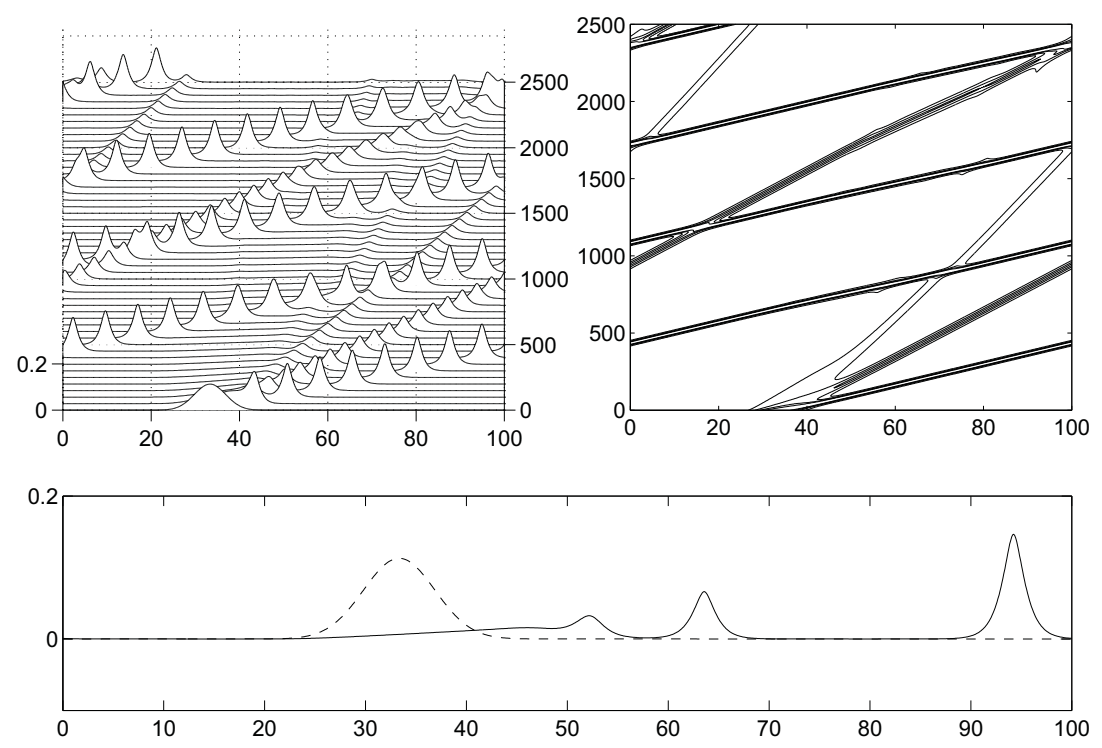

Рис. 1. Пульсонные решения (1.2) уравнения (1.1) возникают из гауссиана, имеющего единичную площадь, ширину $\sigma=5$ и центр около точки $x=33$ в области длины $L=100$ с периодическими граничными условиями. Наиболее быстрый пульсон пересекает область четыре раза и упруго сталкивается с более медленными пульсонами.

Хорошо известно, что интегрируемые УЧП, такие как уравнение КдФ или уравнение Камасса-Холма [2], обладают парой согласованных гамильтоновых структур, которые в совокупности задают оператор рекурсии, порождающий бесконечно много симметрий старших порядков. Мы безуспешно попытались найти аналогичную бигамильтонову структуру для уравнения пятого порядка (1.1); вследствие этого мы убеждены, что это уравнение допускает только одну гамильтонову структуру (1.5).

Как уравнение Камасса-Холма (1.9), так и новое интегрируемое уравнение (2.2), вычлененное Дегасперисом и Прочези [5], обладают слабым свойством Пенлеве (см. [9]), в 
котором в обшем решении допускаются алгебраические ветвления. В литературе имеются многочисленные примеры систем, интегрируемых по Лиувиллю в случае конечного числа измерений [17], и УЧП, интегрируемых с помошью пары Лакса [12], в решениях которых наблюдается это свойство. В случае эволюционных уравнений с помощью преобразований типа годографа в этом случае удается восстановить сильное свойство Пенлеве [11], [12], и сходным образом в работах [3], [6] мы применяли обратные преобразования к неэволюционным уравнениям (1.9) и (2.2). Чтобы проделать анализ Пенлеве в случае уравнения (1.1), оказалось удобным применить обратное преобразование, которое устраняет ветвление в ведушем порядке выражения для решения, но дальнейший анализ редукций этого уравнения к разложению бегуших волн показал, что алгебраическое ветвление все равно остается в условиях главного равновесия ввиду наличия резонансов при полуцелых значениях степенных показателей. Более того, условие резонанса оказывается нарушенным как в главных, так и в неглавных условиях равновесия. Тем самым после преобразования система перестает удовлетворять даже слабому варианту теста Пенлеве.

Мы также применили тест на интегрируемость, который, кажется, вышел из моды в современной науке, а именно метод расширенной алгебры, предложенный Уолквистом и Эстебруком [15]. При сделанных нами некоторых достаточно простых предположениях было найдено, что уравнение (1.1) не допускает никакой пары Лакса полиномиального вида.

Мы не можем ожидать, что $N$-частичная пульсонная динамическая система будет интегрируема при произвольном значении $N>2$, поскольку это означало бы наличие бесконечномерного интегрируемого подмножества в множестве решений неинтегрируемого УЧП. Тем не менее было бы интересно представить аналитическое объяснение солитоноподобного поведения пульсонных решений и их численно проверенной стабильности, которая ясно видна на рис. 2. Поэтому понимание свойств стабильности пульсонных решений требует дальнейших аналитических и численных исследований.
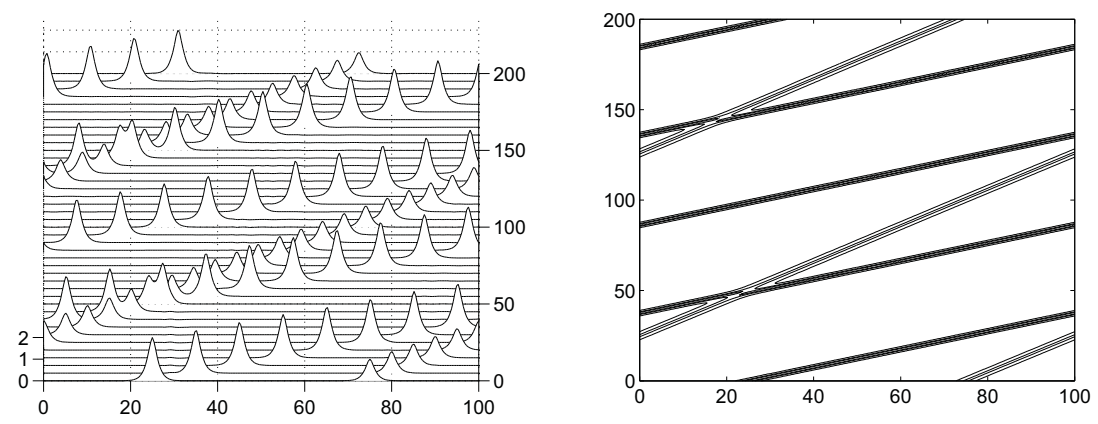

Рис. 2. Столкновения пульсонных решений уравнения (1.1) при набегании сзади с начальными позициями в точках $x=25$ и $x=75$; скорость более быстрого пульсона в два раза больше скорости медленного. При таком отношении скоростей оба соударения приводят к фазовому сдвигу вправо для более быстрой пространственно-временной траектории; более медленная траектория не испытывает фазового сдвига. 
Благодарности. Авторы благодарны М. Стэли за предоставленные рисунки. Один из авторов (А. Х.) признателен CR Barber Trust (Institute of Physics), IMS (University of Kent) и организаторам коференции NEEDS 2002 за финансовую поддержку. Авторы благодарят за гостеприимство Центр математических исследований университета г. Ворвик в период семинара "Геометрия, симметрия и механика II", где эта работа была завершена.

\section{Список литературы}

[1] O. Fringer, D. D. Holm. Phys. D. 2001. V. 150. P. 237-263.

[2] R. Camassa, D. D. Holm. Phys. Rev. Lett. 1993. V. 71. P. 1661-1664; R. Camassa, D. D. Holm, J. M. Hyman. Adv. Appl. Mech. 1994. V. 31. P. 1-33.

[3] A. Degasperis, D. D Holm, A. N. W. Hone. A class of equations with peakon and pulson solutions; in preparation (2002).

[4] D. D. Holm, M. F. Staley. Wave structures and nonlinear balances in a family of $1+1$ evolutionary PDEs. nlinCD/0202059.

[5] A. Degasperis, M. Procesi. Asymptotic integrability. In: Symmetry and Perturbation Theory. Eds. A. Degasperis, G. Gaeta. Singapore: World Scientific, 1999. P. 23-37.

[6] А. Дегасперис, Д. Д. Холм, А. Н. В. Хон. ТМФ. 2002. Т. 133. С. 170-183.

[7] A. V. Mikhailov, V. S. Novikov. J. Phys. A. 2002. V. 35. P. 4775-4790.

[8] H. R. Dullin, G. A. Gottwald, D. D. Holm. Phys. Rev. Lett. 2002. V. 87. P. 194501-194504.

[9] A. Ramani, B. Dorizzi, B. Grammaticos. Phys. Rev. Lett. 1982. V. 49. P. 1538-1541.

[10] J. G. Kingston, C. Rogers. Phys. Lett. A. 1982. V. 92. P. 261-264.

[11] P. A. Clarkson, A. S. Fokas, M. J. Ablowitz. SIAM J. Appl. Math. 1989. V. 49. P. 1188-1209.

[12] A. N. W. Hone. Phys. Lett. A. 1998. V. 249. P. 46-54.

[13] M. J. Ablowitz, A. Ramani, H. Segur. Lett. Nuovo Cimento. 1978. V. 23. P. 333-338.

[14] А. В. Михайлов, А. Б. Шабат, Р. И. Ямилов. УМН. 1987. Т. 42. С. 3-53.

[15] H. D. Wahlquist, F. B. Estabrook. J. Math. Phys. 1975. V. 16. P. 1-7; 1976. V. 17. P. 1293-1297.

[16] A. P. Fordy. Prolongation structures of nonlinear evolution equations. In: Soliton Theory: A Survey of Results. Ed. A. P. Fordy. Manchester: Manchester Univ. Press, 1990. P. 403-425.

[17] S. Abenda, Y. Fedorov. Acta Appl. Math. 2000. V. 60. P. 137-178. 